University of Nebraska - Lincoln

DigitalCommons@University of Nebraska - Lincoln

Faculty Publications from the Department of Electrical \& Computer Engineering, Department Electrical and Computer Engineering

3-31-1997

\title{
Infrared ellipsometry on hexagonal and cubic boron nitride thin films
}

E. Franke

Institute of Surface Modification, D-04303 Leipzig, Germany, efranke3@unl.edu

H. Neumann

Institute of Surface Modification, Leipzig, Germany

Mathias Schubert

University of Nebraska-Lincoln, mschubert4@unl.edu

T. E. Tiwald

University of Nebraska-Lincoln

John A. Woollam

University of Nebraska-Lincoln, jwoollam1@unl.edu

See next page for additional authors

Follow this and additional works at: https://digitalcommons.unl.edu/electricalengineeringfacpub

Part of the Electrical and Computer Engineering Commons

Franke, E.; Neumann, H.; Schubert, Mathias; Tiwald, T. E.; Woollam, John A.; and Hahn, J., "Infrared ellipsometry on hexagonal and cubic boron nitride thin films" (1997). Faculty Publications from the Department of Electrical and Computer Engineering. 47.

https://digitalcommons.unl.edu/electricalengineeringfacpub/47

This Article is brought to you for free and open access by the Electrical \& Computer Engineering, Department of at DigitalCommons@University of Nebraska - Lincoln. It has been accepted for inclusion in Faculty Publications from the Department of Electrical and Computer Engineering by an authorized administrator of DigitalCommons@University of Nebraska - Lincoln. 


\section{Authors}

E. Franke, H. Neumann, Mathias Schubert, T. E. Tiwald, John A. Woollam, and J. Hahn 


\title{
Infrared ellipsometry on hexagonal and cubic boron nitride thin films
}

\author{
E. Franke ${ }^{\mathrm{a})}$ and $\mathrm{H}$. Neumann \\ Institute of Surface Modification, D-04303 Leipzig, Germany \\ M. Schubert \\ Faculty of Physics and Geoscience, University of Leipzig, D-04103 Leipzig, Germany \\ T. E. Tiwald and J. A. Woollam \\ Center for Microelectronic and Optical Materials Research, and Department of Electrical Engineering, \\ University of Nebraska-Lincoln, Lincoln, Nebraska 68588 \\ J. Hahn \\ Institute of Physics, Technical University Chemnitz-Zwickau, D-09107 Chemnitz, Germany
}

(Received 9 December 1996; accepted for publication 28 January 1997)

Infrared spectroscopic ellipsometry (IRSE) over the wavelength range from 700 to $3000 \mathrm{~cm}^{-1}$ has been used to study and distinguish the microstructure of polycrystalline hexagonal and cubic boron nitride thin films deposited by magnetron sputtering onto (100) silicon. The IRSE data are sensitive to the thin-film layer structure, phase composition, and average grain $c$-axes orientations of the hexagonal phase. We determine the amount of cubic material in high cubic boron nitride content thin films from the infrared optical dielectric function using an effective medium approach.

(C) 1997 American Institute of Physics. [S0003-6951(97)03813-8]

High-resolution transmission electron microscopy (HRTEM) and selected area electron diffraction (SAD), ${ }^{1,2}$ have been widely used to investigate the microstructure of polycrystalline boron nitride (BN) thin films deposited by various chemical and physical vapor deposition methods. ${ }^{3,4}$ The hexagonal BN ( $h$-BN, $s p^{2}$-bonded) phase is deposited in nearly pure-phase polycrystalline thin films, with the texture of the $h$-BN grains depending on the growth conditions. As a result, the thin films possess different anisotropic polarized visible light reflectance, as recently discussed for BN thin films grown by magnetron sputtering. ${ }^{5}$ The high cubic $\mathrm{BN}\left(c\right.$-BN, $s p^{3}$-bonded) content thin films consist of singlephase nanocrystalline $c$-BN grains with $s p^{2}$-bonded material ( $h$-BN) at the grain boundaries. ${ }^{2}$ The $c$-BN thin layers are grown after the deposition of a noncubic nucleation layer.

The BN lattice resonance absorption behavior is known from bulk investigations. ${ }^{6-9}$ Geick et al. ${ }^{6}$ ( $h$-BN) report the out-of-plane $(E \| c)$ and in-plane $(E \perp c)$ transverse (TO) and longitudinal (LO) optical frequencies, $\omega_{\mathrm{TO} \|}=783 \mathrm{~cm}^{-1}$, $\omega_{\mathrm{LO} \|}=828 \mathrm{~cm}^{-1}, \quad \omega_{\mathrm{TO} \perp}=1367 \mathrm{~cm}^{-1}, \quad$ and $\omega_{\mathrm{LO} \perp}$ $=1610 \mathrm{~cm}^{-1}$, respectively. Gielisse et al. ${ }^{7}(c-\mathrm{BN})$ derived $\omega_{\mathrm{TO} c}=1065 \mathrm{~cm}^{-1}$ and $\omega_{\mathrm{LO} c}=1340 \mathrm{~cm}^{-1}$, while Eremets et al. ${ }^{8}$ obtained $\omega_{\mathrm{TO} c}=1056 \mathrm{~cm}^{-1}$ and $\omega_{\mathrm{LO} c}=1305 \mathrm{~cm}^{-1}$. The gap between $\omega_{\mathrm{TO} \perp}$ and $\omega_{\mathrm{TO} \|}$ is indicative of the optical anisotropy of $h$-BN. Because of the easily distinguishable $h$-BN and $c$-BN infrared lattice resonance absorption behavior, infrared spectroscopy (IRS) is widely used to determine the BN thin-film phase. $^{3}$ Oblique angle of incidence $p$-polarized IRS is sensitive to the thin-film LO mode frequency as well, if the film thickness is less than a fraction of the incident light wavelength. This is known as the Berreman effect $^{10}$ and has been demonstrated recently for BN thin films. ${ }^{11}$

However, the thin-film infrared reflectance is a function of the microstructure, and no attempt has been reported on a

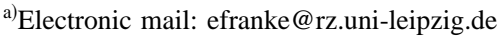

quantitative thin-film analysis. As well, none of the above methods allows for the determination of the amount of $h$-BN within the high $c$-BN content films. A simple macroscopic and nondestructive characterization of the phase and microstructure of BN thin films is crucial for the future development of this field.

The present work reports on the application of infrared spectroscopic ellipsometry (IRSE) in order to simultaneously determine the phase and microstructure of BN thin films. We describe a model which allows for the interpretation of the IRSE data which depends on the thin-film layer structure, phase composition, and average grain $c$-axes orientations of the $h$-BN phase. An effective medium approach is used to determine the amount of $h$-BN material within the high $c$-BN content thin films. Compared to HRTEM investigations the microstructure dependent information obtained by IRSE is characteristic of general thin-film properties.

The BN thin films were deposited on (100) silicon by magnetron sputtering at various substrate bias voltages $U_{B}$, resulting in polycrystalline and nearly pure $h-\mathrm{BN}$ or $c-\mathrm{BN}$ thin films. ${ }^{12}$ The samples were investigated by HRTEM and SAD. A noncubic nucleation layer was observed between the substrate and the high $c$-BN content layer in the $c$-BN thin films. The grain $c$-axes orientations within the $h$-BN layers depend on the growth conditions $\left(U_{B}\right)$. Spectroscopic ellipsometry in the visible spectral range was used to determine the thin-film refractive indices, and it was found that the microscopic $c$ axes of the $h$-BN grains possess an average inclination angle $\Theta .^{5}$ The light beam contains information from numerous grains and averages their inclinations over the range of in-plane orientations, thereby mapping the $c$ axes onto the outer shell of a cone. The cone angle $\Theta$ then describes the average orientation of the grain $c$ axes. The resulting $h$-BN dielectric function tensor was experimentally found to be independent of any in-plane sample orientation dependence.

IRSE measurements were carried out in a wave number 

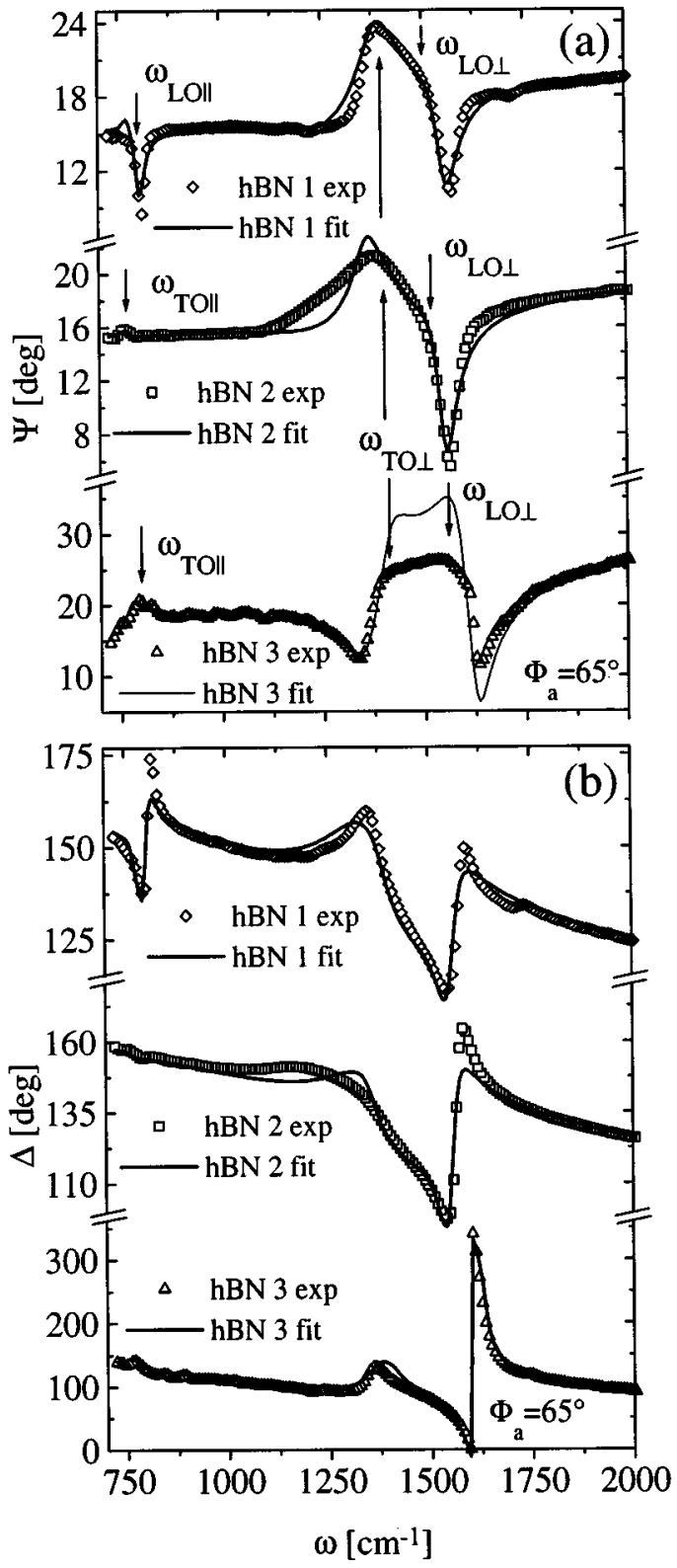

FIG. 1. The $h$-BN experimental and generated data $\left(\Phi_{a}=65^{\circ}\right.$; (a) $\Psi$, (b) $\left.\Delta\right)$. The various $\Psi$ spectra are due to the different grain $c$ axes orientations.

range between 700 and $3000 \mathrm{~cm}^{-1}$ at multiple angles of incidence $\left(\Phi_{a}=55^{\circ}-75^{\circ}\right)$. We utilized a rotating-polarizer, rotating-compensator, Fourier-transform based spectroscopic ellipsometer.

Ellipsometry determines phase and absolute value of the complex reflectance ratio $\rho=R_{p} / R_{s}=\tan \Psi \exp (i \Delta)$, where $R_{p}$ and $R_{s}$ are the complex reflectance coefficients of light polarized perpendicular $(s)$ and parallel $(p)$ to the plane of incidence. ${ }^{13}$ In general, the spectroscopic data must be numerically fit. For this purpose, a model must be assumed and $\Psi$ and $\Delta$ are generated and compared with the measured data for the same wave number and incidence set.

Ellipsometric data were analyzed by modeling each lattice absorption as $c$-BN or $h$-BN according to Geick et al. ${ }^{6}$ and Ermets et al. ${ }^{8}$ The optical frequency dielectric function can be written as follows:
TABLE I. Best sample model phase and microstructure parameters.

\begin{tabular}{|c|c|c|c|c|}
\hline $\begin{array}{c}\text { Sample } \\
\left(U_{B}[\mathrm{~V}]\right)\end{array}$ & $\begin{array}{c}\text { Top layer } \\
\text { bottom layer }\end{array}$ & $f_{c}[\%]$ & $\begin{array}{l}d_{1}[\mathrm{~nm}] \\
d_{2}[\mathrm{~nm}]\end{array}$ & $\begin{array}{l}\Theta_{1}\left[^{\circ}\right] \\
\Theta_{2}\left[^{\circ}\right]\end{array}$ \\
\hline$c \mathrm{BN} 1$ & $c / h-\mathrm{BN}$ & $83 \pm 5$ & $94 \pm 2$ & $54.7^{\mathrm{b}}$ \\
\hline$(-150)$ & $h-\mathrm{BN}$ & $\ldots$ & $3.5 \pm 1$ & $90 \pm 5$ \\
\hline$c \mathrm{BN} 2$ & $c / h-\mathrm{BN}$ & $75 \pm 5$ & $120 \pm 3$ & $54.7^{\mathrm{b}}$ \\
\hline$(-150)$ & $h$-BN & $\ldots$ & $2.5 \pm 1$ & $90 \pm 5$ \\
\hline$c \mathrm{BN} 3$ & $c / h-\mathrm{BN}$ & $70 \pm 5$ & $131 \pm 3$ & $54.7^{\mathrm{b}}$ \\
\hline$(-150)$ & $h$-BN & $\cdots$ & $24 \pm 1$ & $70 \pm 5$ \\
\hline$h \mathrm{BN} 1$ & $h-\mathrm{BN}$ & $\ldots$ & $178 \pm 4$ & $34 \pm 5$ \\
\hline$(+20)^{\mathrm{a}}$ & $h-\mathrm{BN}$ & $\ldots$ & $100 \pm 2$ & $54.7^{\mathrm{b}}$ \\
\hline$h \mathrm{BN} 2$ & $h-\mathrm{BN}$ & $\ldots$ & $256 \pm 5$ & $54.7^{\mathrm{b}}$ \\
\hline$(-70)$ & $\cdots$ & $\cdots$ & $\cdots$ & $\cdots$ \\
\hline$h \mathrm{BN} 3$ & $h-\mathrm{BN}$ & $\cdots$ & $520 \pm 8$ & $90 \pm 5$ \\
\hline$(-30)$ & $\cdots$ & $\cdots$ & $\cdots$ & $\cdots$ \\
\hline
\end{tabular}

${ }^{\mathrm{a}}$ Floating potential, no supplied substrate voltage.

${ }^{\mathrm{b}}$ Isotropically averaged $h$-BN.

$$
\epsilon_{j}=\epsilon_{0 j} \omega_{\mathrm{TO} j}^{2} / \omega_{\mathrm{LO} j}^{2}+\epsilon_{0 j} \omega_{\mathrm{TO} j}^{2} \frac{1-\omega_{\mathrm{TO} j}^{2} / \omega_{\mathrm{LO} j}^{2}}{\omega_{\mathrm{TO} j}^{2}-\omega^{2}-i \omega \Gamma_{j}},
$$

where $\epsilon_{0}$ is the static dielectric constant, $\omega_{\mathrm{TO}}$ and $\omega_{\mathrm{LO}}$ are the transverse and longitudinal optical frequencies, and $\Gamma$ is the lattice resonance broadening parameter. The index $j$ refers to the in-plane " $\perp$," out-of-plane " $\|$ " $(h$-BN), and $c$-BN " $c$ ", optical lattice absorptions.

The $h$-BN thin-film dielectric functions for polarization parallel $\left(\epsilon_{\mathrm{o}, \mathrm{eff}}\right)$ and perpendicular $\left(\epsilon_{e, \text { eff }}\right)$ to the film normal average between $\epsilon_{\perp}$ and $\epsilon_{\|}$, according to Eqs. (1) and (2) in Ref. 5

$$
\begin{aligned}
& \epsilon_{\mathrm{o}, \mathrm{eff}}=\left\{(3+\cos 2 \Theta) \epsilon_{\perp}+(1-\cos 2 \Theta) \epsilon_{\|}\right\} / 4, \\
& \epsilon_{e, \mathrm{eff}}=\left(\epsilon_{\|} \cos ^{2} \Theta\right)+\left(\epsilon_{\perp} \sin ^{2} \Theta\right) .
\end{aligned}
$$

The thin-film TO mode frequencies are the poles of Eq. (2a). The LO mode frequencies are represented by the roots of Eq. (2b) and depend on the cone angle $\Theta$. A more detailed discussion of the microstructure dependent $h$-BN thin-film anisotropic dielectric function will be presented elsewhere. ${ }^{14}$

The dielectric function $\epsilon$ for the high $c$-BN content thin films can be described by the Bruggemann effective medium approximation (EMA). We assume randomly oriented $s p^{2}$-bonded material at the grain boundaries. This isotropically averaged $h$-BN is described through Eqs. (2) at $\cos \Theta$ $=1 / \sqrt{3}$, where $\epsilon_{h}=\epsilon_{e, \text { eff }}=\epsilon_{\mathrm{o}, \text { eff }}=\epsilon_{\|} / 3+2 \epsilon_{\perp} / 3$. We obtain $\epsilon$ from

$$
f_{c}=\left(\epsilon_{c}-\epsilon\right) /\left(\epsilon_{c}+2 \epsilon\right)+f_{h}\left(\epsilon_{h}-\epsilon\right) /\left(\epsilon_{h}+2 \epsilon\right)=0,
$$

where $\epsilon_{c}, \epsilon_{h}, f_{c}$, and $f_{h}$ are the dielectric functions and the volume fraction of $c$-BN and $h$-BN, respectively. ${ }^{15}$

Figure 1 shows generated and experimental data $(\Psi, \Delta)$ from three different $h$-BN samples (Table I). Data from one angle of incidence $\left(\Phi_{a}=65^{\circ}\right)$ are presented only, to avoid confusion between the otherwise multiple plots.The generated data were obtained from the best fit for the $h$-BN dielectric function parameters $\left(\epsilon_{0 \perp}, \omega_{\mathrm{TO} \perp}, \omega_{\mathrm{LO} \perp}, \Gamma_{\perp}, \epsilon_{0 \|}, \omega_{\mathrm{TO} \|}, \omega_{\mathrm{LO} \|}, \Gamma_{\|}\right)$, the cone angle $\Theta$, and layer thickness $d$. A two-layer model was used to predict the data from sample $h \mathrm{BN} 1$ according to the HRTEM and SAD investigations allowing for each layer cone angle, thickness, and broadening parameters separately. 

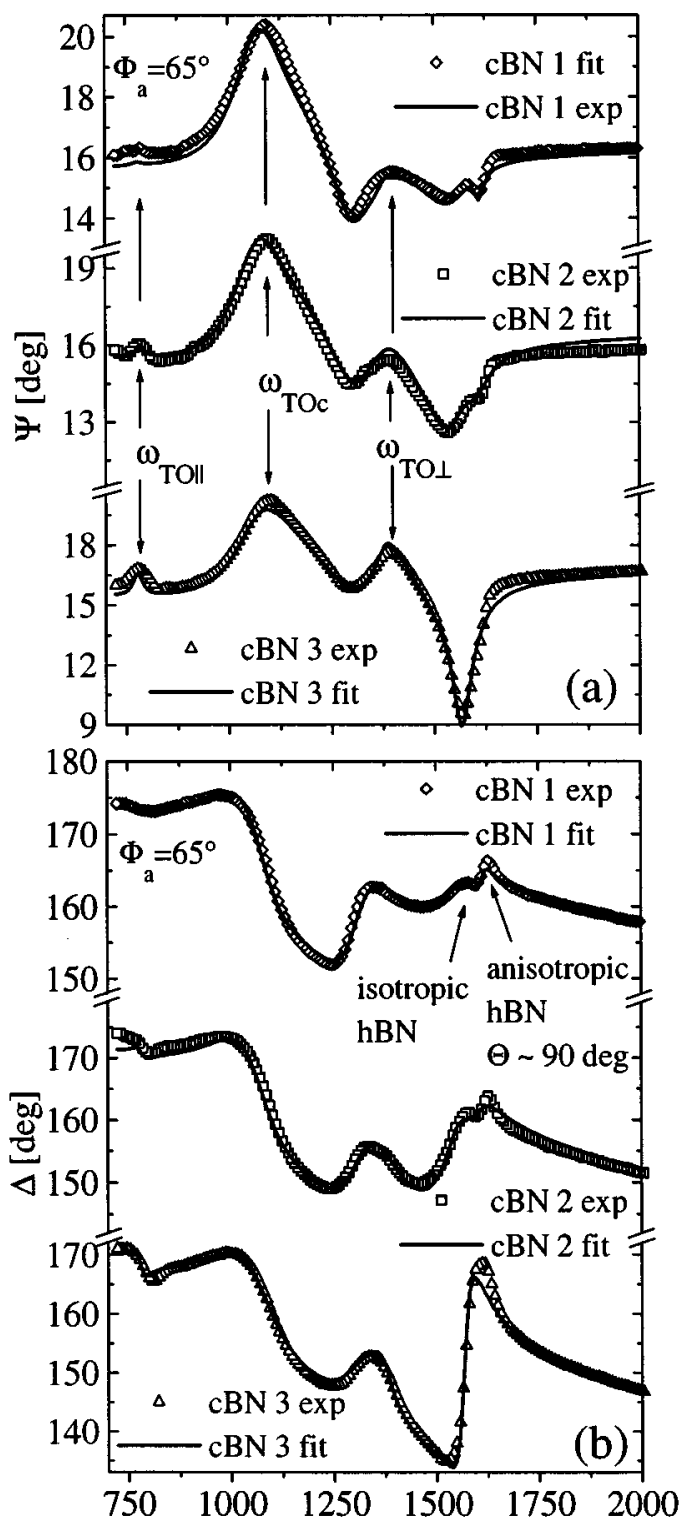

$\omega\left[\mathrm{cm}^{-1}\right]$

FIG. 2. Same as Fig. 1 for $c$-BN $\left(\Phi_{a}=65^{\circ}\right.$; (a) $\Psi$, (b) $\left.\Delta\right)$. The various spectra are due mainly to the different $c$-BN contents within the thin films. The double peak structure at $\omega \sim 1600 \mathrm{~cm}^{-1}$ is due to two different $h$-BN in-plane LO modes excited within the thin films (isotropically averaged $h$-BN: $\quad \omega_{\mathrm{LO} \perp} \sim 1500 \mathrm{~cm}^{-1} ; \quad$ anisotropic $\quad h$-BN $\quad\left(\Theta \sim 90^{\circ}\right): \quad \omega_{\mathrm{LO} \perp}$ $\left.\sim 1600 \mathrm{~cm}^{-1}\right)$.

The different spectra of the three samples are attributed to different $h$-BN grain $c$-axes orientations. The in-plane TO resonance frequency $\omega_{\mathrm{TO} \perp}$ is identical within all samples, whereas the apparent $\mathrm{LO}$ mode frequency $\omega_{\mathrm{LO} \perp}$ shifts as a function of $\Theta$. This can be demonstrated through Eqs. (2). ${ }^{14}$ The large broadening of the $\omega_{\mathrm{TO} \perp}$ peak in sample $h \mathrm{BN} 2$ is explained by very small grain sizes. The out-of-plane lattice resonance behavior of the samples is affected by their microstructure as well. Again, the TO mode frequency $\omega_{\mathrm{TO}}$ is one of the poles of $\epsilon_{\mathrm{o}, \text { eff }}$ and independent of $\Theta$. The LO mode frequency $\omega_{\mathrm{LO}}$ is a function of $\Theta$ but difficult to observe if the grain $c$ axes are bent far away from the sample normal. A strong LO phonon mode resonance is excited in sample $h \mathrm{BN} 1\left(\Theta \sim 35^{\circ}\right)$. Sample $h \mathrm{BN} 2\left(\Theta \sim 54.7^{\circ}\right)$ consists of iso- tropically averaged $h$-BN. Here the out-of-plane lattice resonance absorption features virtually disappear. This is not indicative of the existence of a new modification but rather a demonstration of the validity of Eqs. (2), and inherent to the dielectric function model discussed above. The lattice resonance parameters obtained from the best fit between the data and the model are similar for all samples $\left(\omega_{\mathrm{TO} \|}\right.$ $\sim 775 \mathrm{~cm}^{-1}, \omega_{\mathrm{LO} \|} \sim 820 \mathrm{~cm}^{-1}, \omega_{\mathrm{TO} \perp} \sim 1400 \mathrm{~cm}^{-1}, \omega_{\mathrm{LO} \perp}$ $\sim 1585 \mathrm{~cm}^{-1}$ ) and agree with bulk values. The remaining parameters are summarized in Table I.

Figure 2 shows generated and experimental data $(\Psi, \Delta)$ from three different $c$-BN samples (Table I). A two-layer model was used to account for the $h$-BN nucleation layer. The generated data were obtained from the best fit for the $c$-BN dielectric function parameters $\left(\epsilon_{0 c}, \omega_{\mathrm{TO} c}, \omega_{\mathrm{LO} c}\right.$, $\Gamma_{C}$ ), the $h$-BN parameters as described above, the layer thicknesses $d_{1}$ and $d_{2}$, the $c$-BN volume fraction $f_{c}=1$ $-f_{h}$, and the nucleation layer cone angle $\Theta$. The volume fractions $f_{c}$ obtained here are about $5 \%$ less than the $c$-BN concentration determined from the peak height ratio using IRS data from the same samples. ${ }^{3}$ The double peak structures in $\Psi, \Delta$ at $\omega \sim 1600 \mathrm{~cm}^{-1}$ reveal the nucleation layer grain $c$-axes orientations. ${ }^{14}$ The differences between spectra on all samples are due mainly to different volume fractions of $c$-BN within the thin films. The pronounced $c$-BN TO mode is indicated by arrows, together with the $h$-BN TO mode frequencies which are still present. The $c$-BN lattice resonance parameters obtained from the best fit are unique within all samples $\left(\omega_{\mathrm{TO} c}=1070 \mathrm{~cm}^{-1}, \omega_{\mathrm{LO} c}=1300 \mathrm{~cm}^{-1}\right)$ and agree with bulk data. The microstructure dependent fit parameters are given in Table I as well.

The authors wish to acknowledge Daniel W. Thompson and Mick DeVries for technical support during the experiments. We thank the Center for Microelectronic and Optical Materials Research for financial support during part of the work.

${ }^{1}$ D. J. Kester, K. S. Ailey, D. J. Lichtenwalner, and R. F. Davis, J. Vac. Sci. Technol. A 12, 3074 (1994).

${ }^{2}$ W. L. Zhou, Y. Ikuhara, M. Murakawa, S. Watanabe, and T. Suzuki, Appl. Phys. Lett. 66, 2490 (1995).

${ }^{3}$ T. A. Friedmann, P. B. Mirkarimi, D. L. Medlin, K. F. McCarty, E. J. Klaus, D. R. Boehme, H. A. Johnson, M. J. Mills, and D. K. Ottesen, J. Appl. Phys. 76, 3088 (1994).

${ }^{4}$ S. Reinke, M. Kuhr, W. Kulisch, and R. Kassing, Diam. Relat. Mater. 4, 272 (1995).

${ }^{5}$ M. Schubert, B. Rheinländer, E. Franke, H. Neumann, J. Hahn, M. Röder, and R. Richter, Appl. Phys. Lett. (in press).

${ }^{6}$ R. Geick, C. H. Perry, and G. Rupprecht, Phys. Rev. 146, 543 (1966)

${ }^{7}$ P. J. Gielisse, S. S. Mitra, J. N. Plendl, R. D. Griffis, L. C. Mannsur, R. Marshall, and E. A. Pascoe, Phys. Rev. 155, 1039 (1967).

${ }^{8}$ M. I. Eremets, M. Gauthier, A. Polian, J. C. Chervin, J. M. Besson, G. A. Dubitskii, and Ye. Ye. Semenova, Phys. Rev. B 52, 8854 (1995).

${ }^{9}$ D. M. Hoffman, G. L. Doll, and P. C. Eklund, Phys. Rev. 30, 6051 (1984).

${ }^{10}$ D. W. Berreman, Phys. Rev. 130, 2193 (1963).

${ }^{11}$ M. F. Plass, W. Fukarek, S. Mändl, and W. Möller, Appl. Phys. Lett. 69, 46 (1996).

${ }^{12}$ J. Hahn, M. Friedrich, R. Pintaske, M. Schaller, N. Kahl, D. R. T. Zahn, and R. Richter, Diam. Relat. Mater. 5, 1103 (1996).

${ }^{13}$ R. M. A. Azzam and N. M. Bashara, Ellipsometry and Polarized Light (North-Holland, Amsterdam, 1984).

${ }^{14}$ M. Schubert, B. Rheinländer, E. Franke, H. Neumann, T. E. Tiwald, and J. A. Woollam, J. Hahn (unpublished).

${ }^{15}$ D. E. Aspens and J. B. Theeten, Phys. Rev. B 20, 3292 (1979). 